\title{
Detection of coatings and measurement of coating thickness on technical substrates using surface acoustic waves in a waveguide configuration
}

\author{
Gerhard Lindner, Martin Schmitt, Katrin Schmidt, Hendrik Faustmann, Sandro Krempel and \\ Josefine Schubert \\ Institute of Sensor and Actuator Technology, Coburg University of Applied Sciences \\ D-96450 Coburg, Germany
}

\begin{abstract}
Surface acoustic waves turned out to be very sensitive to deposition layers on their propagation path on the solid substrate, which both influence the propagation velocity of the waves and their amplitude. In our approach, Lamb waves are excited on arbitrary technical substrates like metals, glass or plastics with a thickness of not more than the acoustic wavelength via interdigital piezoelectric transducers attached to the backside of the substrate. The performance of this approach was demonstrated with simple delay lines made of thin glass plates and with acoustic waveguides formed by parallel glass plates or a plastic tube, which will be covered by plastic films of different thicknesses on the inner surface. This concept allows an on-line monitoring of coverage layer formation on the inner wall of a liquid-filled conduit and the measurement of the layer thickness from outside. A sensitivity in the order of $1 \mathrm{~ns}$ time shift of the receiver signal per $\mu \mathrm{m}$ layer thickness has been achieved so far, which depends, however, on the type of material both of the substrate and the deposition layer.
\end{abstract}

\section{Introduction}

The propagation of surface acoustic waves on solid substrates turned out to be highly sensitive to the deposition of coatings on their propagation path thus allowing a characterization of such coatings via corresponding changes of the propagation behaviour of the surface acoustic waves [1-4]. In general, both the velocity as well as the attenuation of surface acoustic waves was affected by such coatings depending of the material properties such as elastic parameters, density or the thickness of the coating [4-7]. Different approaches to the excitation and detection of surface acoustic waves have been reported, among them photoacoustic techniques such as laser-generated surface acoustic waves $[4,6]$, acoustic microscopy $[4,8]$ or piezoelectric surface acoustic wave devices equipped with interdigital transducers [5, 7, 9-11]. It has been demonstrated with the latter type of device that very thin films, e.g. Langmuir-Blodgett films of one monolayer (corresponding to a thickness of typically about $3 \mathrm{~nm}$ ) can be detected and individual monolayers can be distinguished [7, 9-11].

In our approach, the deposition of thin coating on the inner wall of a liquid-filled conduit consisting of a non-piezoelectric technical material such as steel, glass or plastics shall be detected from outside using the surface acoustic wave technique. These circumstances, however, exclude the application of the above mentioned technologies. Therefore, following a concept developed for acoustic flow measurements with clamp-on transducers [12], an acoustic waveguide technique based on acoustic surface wave excitation and detection on the wall material of the conduit by means of remotely attached piezoelectric interdigital transducers has been applied: Resulting from the reversible mode conversion of Lamb waves to compression waves at the inner surface [13], the wave pulse follows a zig-zag pathway in the waveguide determined by the Rayleigh angle $[14,15]$. Correspondingly, the transit time of short wave pulses crossing the conduit and the amplitude of the receiver signals depend on the material properties both of the wall material and the liquid [16]. Since the mode conversion at the solid-liquid interface will be influenced by the formation of thin solid layers [17, 18], changes in the receiver signals could be expected in that case, which would allow the remote monitoring of the growth of such deposition layers.

In this contribution, results of preliminary investigations at technical conduits with a focus on polymeric layers will be presented, which allow a rough estimate of the sensitivity of this approach. To this end, different polymer films of rather large thicknesses were deposited (i) on thin glass slides equipped with interdigital transducer thus acting as substrates for Lamb wave transmission in air in order to measure the influence of the deposit on the velocity and the attenuation of the Lamb wave, (ii) on one of the inner surfaces of a water-filled acoustic waveguide formed by the same glass plates in order to check 
the influence on the mode conversion process and (iii) on one of the inner surface of a plastic tube filled with water in order to check the applicability of this concept at technical conduits.

\section{Experimental set-up}

For the measurement of the influence of the deposition of polymer layers on the propagation of Lamb waves, two interdigital transducers made from PZT designed for $1 \mathrm{MHz}$ excitation frequency were attached to one surface of a $1 \mathrm{~mm}$ thick glass plate and layers of different thicknesses were deposited on the opposite surface (Fig. 1a). One of the transducers is excited by narrow-band sine bursts consisting of 5 oscillation periods, whereas one of the other transducers acts as a receiver for the corresponding acoustic wave pulses. Adhesive tape (polypropylene film with acrylic dispersion glue), gelatine and correction fluid with different thicknesses between 30 and $500 \mu \mathrm{m}$ have been applied and both the transit time and the receiver amplitude have been measured with a digital storage oscilloscope. The layer thicknesses were determined by weighting and measuring the area of the layer coverage. The temperature has been kept constant within less than $1{ }^{\circ} \mathrm{C}$ deviations throughout all the measurements.

The influence of the polymer layer deposition on the mode conversion has been investigated with an acoustic waveguide cell formed by two parallel glass plates of the same type as above and filled with carefully degassed distilled water. One of the glass plates could be exchanged against plates loaded with deposition layers and equipped with an exciting transducer as above, but the receiving transducer was attached to the opposite plate (Fig. 1b). Again the transit time and the receiver amplitude were measured as above.

A water-filled glass fibre reinforced plastic tube with $6 \mathrm{~cm}$ diameter and a wall thickness of $3 \mathrm{~mm}$, on which interdigital transducers as described above were attached to the outer surface at different axial positions, and the same measurement procedure was used for the practical tests of the concept with respect to technical conduits (Fig. 1c).

1a)

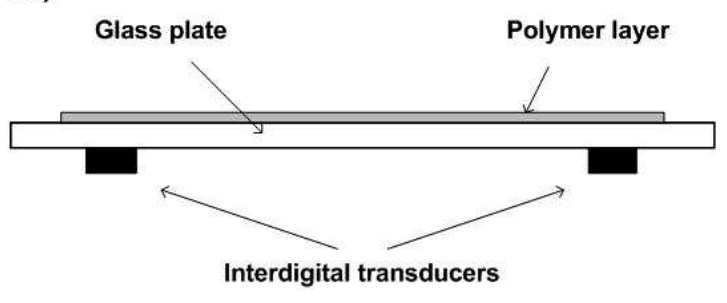

1b)

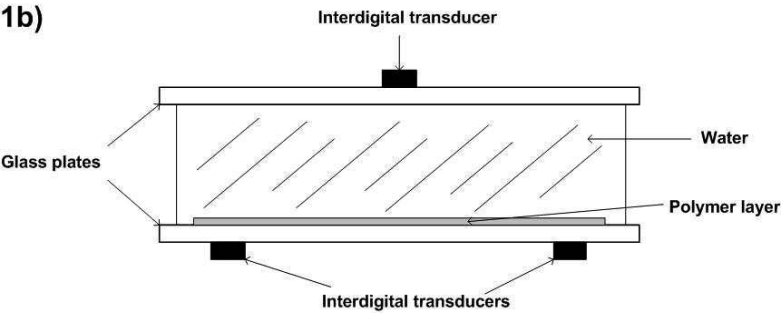

1c)



Fig. 1: Experimental set-ups used in the measurements of acoustic wave transmission.

\section{Results and discussion}

\subsection{Measurements at unloaded transmission lines}

A first group of investigations were performed at glass plates and a plastic tube with different deposition layers according to Fig. 1a and 1c without water loading. From the time shift of the measurement signals from the glass plate shown in Fig. 2 it became apparent that the propagation velocity of the wave pulse - and in this case also the amplitude of the receiver signal - decreases, if a 
layer of organic material is deposited. This was expected for soft layers on a hard substrate like glass. Fig. 3 shows that the influence on the propagation velocity increases monotonously with layer thickness and can be different for various layer materials. Similar observations were recorded for layers on the inner wall of a plastic tube (Fig. 4).

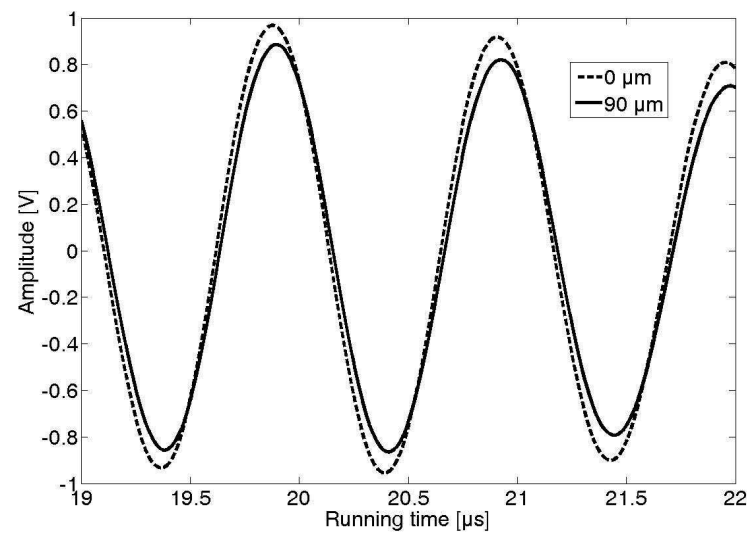

Fig. 2: Receiver signal of a wave pulse on a glass transmission line uncovered and covered with a gelatine layer of $90 \mu \mathrm{m}$ thickness.

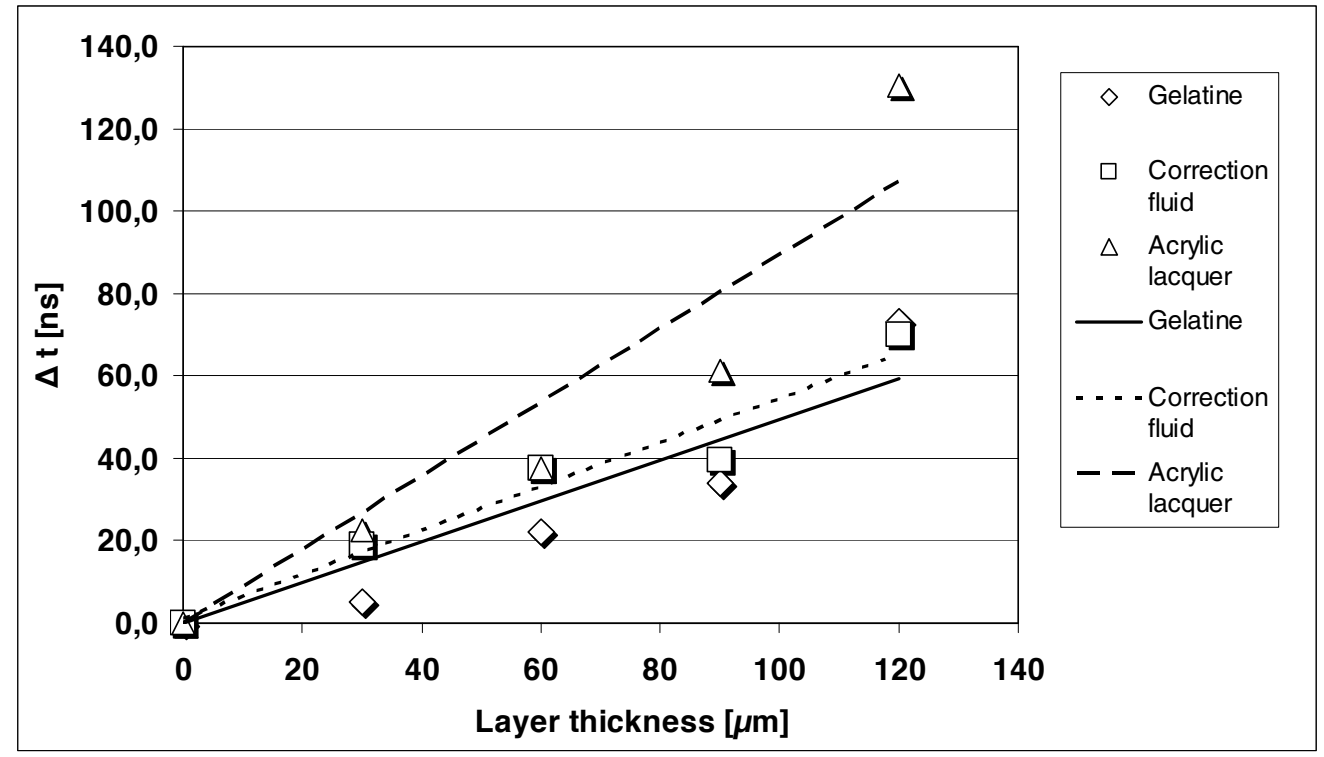

Fig. 3: Relative changes of the transmission time of wave pulses on a glass transmission line covered with layers of gelatine, correction fluid and acrylic lacquer with different thicknesses.
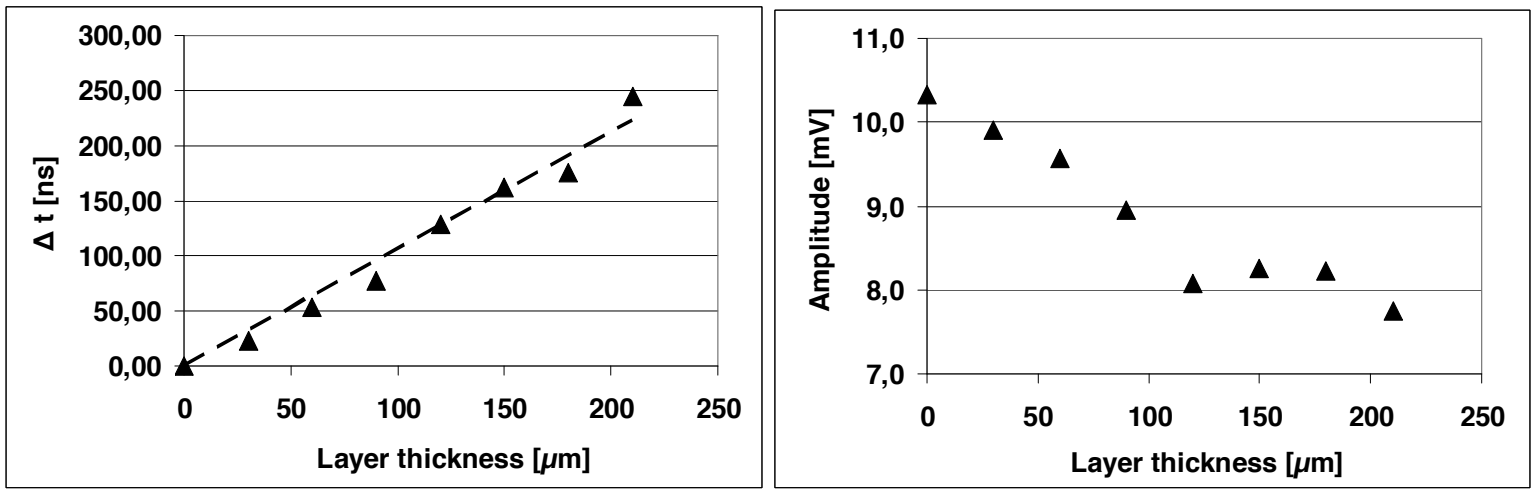

Fig. 4: Relative changes of the transmission time and the receiver amplitude of wave pulses on the inner wall of a plastic tube covered with layers of dried correction fluid with different thicknesses. 


\subsection{Measurements at water-filled acoustic waveguides}

A second group of investigations were performed with water-filled waveguide cells. Different effects were observed with layers on glass: For gelatine layers, for instance, the transit time to the receiver decreased with layer thickness (Fig. 5 and 6), whereas for layers formed by correction fluid a monotonous increase was recorded. Therefore, in the water-filled waveguide configuration, the influence of the layer deposition on the mode conversion and correspondingly on the pathway of the wave pulse between exciting and receiving transducer seems to be more important with respect to the transit time than the propagation velocity of the Lamb waves obtained with the unloaded layered substrate itself.

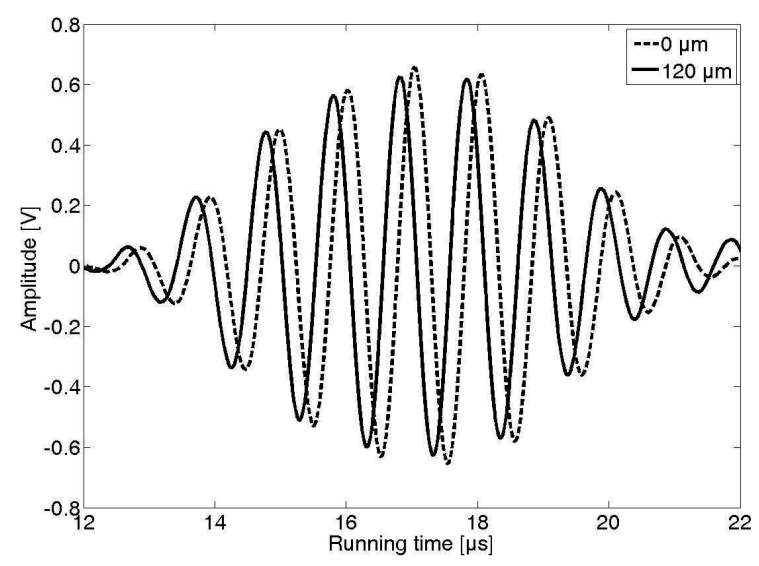

Fig. 5: Receiver signal of a wave pulse in an acoustic waveguide formed of glass plates uncovered and covered on one side with a gelatine layer of $120 \mu \mathrm{m}$ thickness.



Fig. 6: Relative changes of the transmission time of wave pulses in a water-filled acoustic waveguide formed of glass plates covered on one side with gelatine layers with different thicknesses.

These findings were corroborated by results obtained with wave pulses transmitting across the water filling of an acoustic waveguide formed by a plastic tube: Whereas with layers of dried correction fluid deposited on one side of the inner wall the transit time increases with the thickness of the layer, the opposite happens with layers of adhesive film (Fig. 7 and 8). 


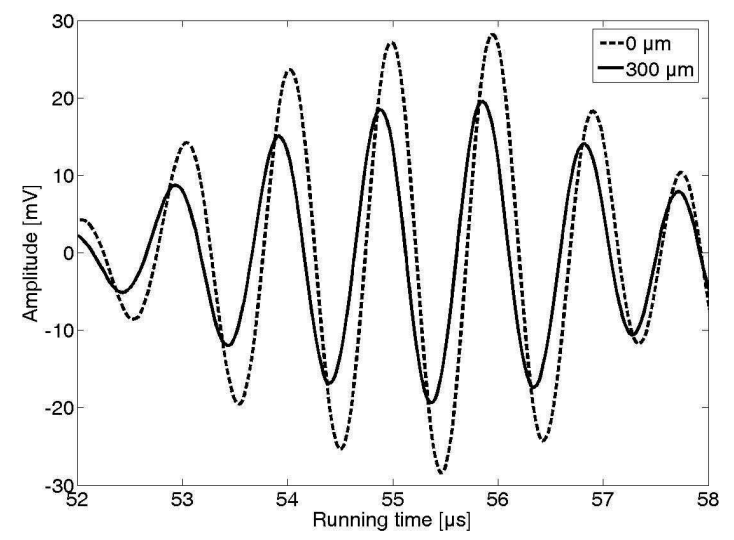

Fig. 7: Receiver signal of a transmitted wave pulse in a water-filled acoustic waveguide formed of a plastic tube uncovered and covered on one side with an adhesive tape layer of $300 \mu \mathrm{m}$ thickness.

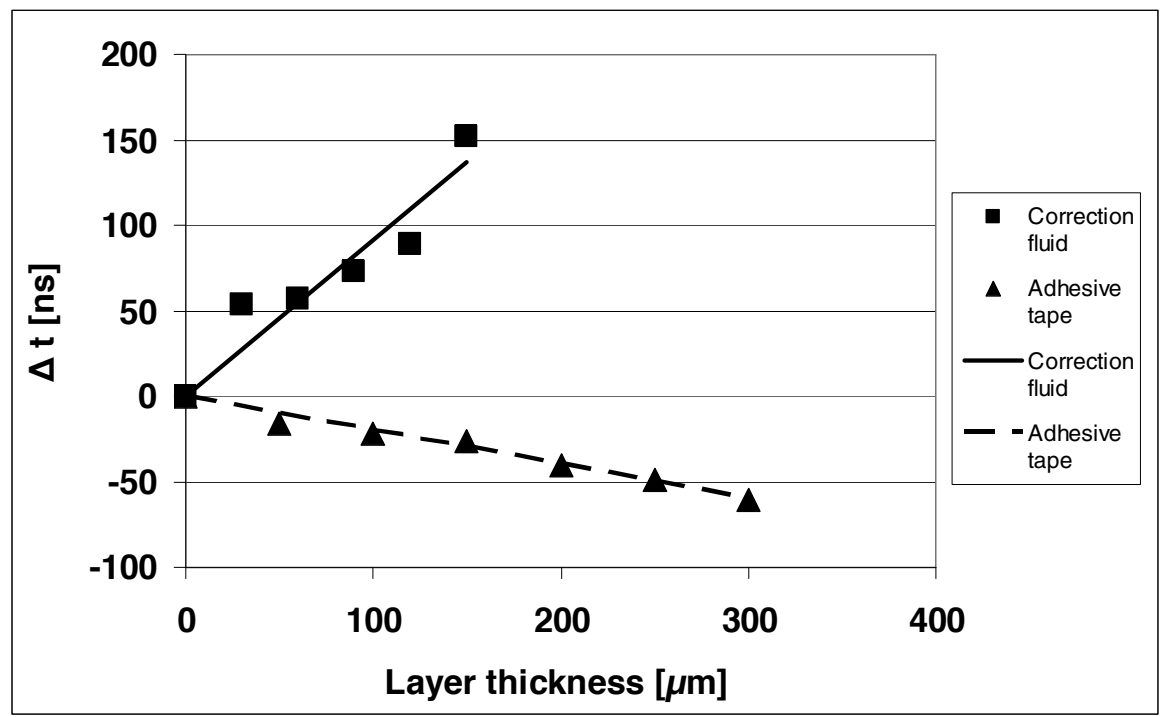

Fig. 8: Relative changes of the transmission time of wave pulses in a water-filled acoustic waveguide formed of plastic tube covered on one side with layers of dried correction fluid and adhesive tape layers with different thicknesses.

Although a comprehensive interpretation of these results needs further investigations, it became apparent that the waveguide configuration is sensitive not only to the deposition of layers on the wall but also to the type of material forming such layers. A sensitivity of about $1 \mathrm{~ns}$ per $\mu \mathrm{m}$ has been achieved so far with layers of dried correction fluid, which is in the order of the desired resolution for many applications. Another quantity, which is influenced by layer deposition, but has not been analyzed so far, is the amplitude of the receiver signal, which in a waveguide cell is not only affected by attenuation of the surface acoustic wave, but also of changes in the acoustic impedance at the interface to the liquid.

\section{Summary and Conclusion}

The results of these investigations demonstrate the feasibility of monitoring layer formation inside conduits by means of external transducers and mode conversion of Lamb waves. The sensitivity obtained so far by measuring the transit time of short wave pulses with a frequency of $1 \mathrm{MHz}$ seems to be sufficient for the detection of layers in the order of a few micrometers; the changes of transit times observed with different layer materials, however, turned out to be strongly dependent on the combination of substrate and layer materials. More detailed studies of the influence of layer deposition on the mode conversion process and of the wave propagation within the waveguides will be necessary 
for an explanation of these findings. In addition, the analysis of the changes of the amplitudes of the receiver signal may provide an additional approach for a further enhancement of the sensitivity.

\section{Acknowledgements}

The authors gratefully acknowledge the support of the BMBF within the program FHprofUnd and they appreciate the cooperation with Fresenius Medical Care Deutschland GmbH.

\section{References}

[1] Krylov, V.V.: Surface properties of solids and surface acoustic waves: Application to chemical sensors and layer characterization, Appl. Phys. A 61 (1995) 229-236.

[2] Ballantine, Jr., D.S., White, R.M., Martin, S.J., Ricco, A.J., Zellers, E.T., Frye, G.C., Wohltjen, H.; Acoustic Wave Sensors: Theory, Design, and Physico-Chemical Applications, Academic Press, San Diego, 1997.

[3] Thompson M., Stone D.C., Thompson Jr., A.: Surface-Launched Acoustic Wave Sensors: Chemical Sensors and Thin-Film Characterization, Wiley, New York, NY, 1997.

[4] Every, A.G.: Measurement of the near-surface elastic properties of solids and thin supported films, Meas. Sci. Technol. 13 (2002) R21-R39.

[5] Wohltjen H., Dessy R.E.: Surface acoustic wave probes for chemical analysis. III. Thermomechanical polymer analyzer, Anal. Chem. 51 (1979) 1470-1475

[6] Schneider, D., Schwarz T., Bradford A.S, Shan Q. and Dewhurst R.J.: Controlling the quality of thin films by surface acoustic waves, Ultrasonics 35 (1997) 345-356.

[7] Cirak, J., Kostial P., Vajda J., Tomcik, P., Barancok, D and Kelesi, L.: Surface acoustic wave propagation in Langmuir-Blodgett layers, Applied Surface Science 108 (1997) 53-57.

[8] Richard Ph., Behrend O., Gremaud G., Kulik A.: Characterization of thin films using generalized Lamb wave dispersion relation, Journal de Physique IV 3 (1993) 2173-2176

[9] Roberts, G.G., Holcroft, B., Barraud A., Richard, J.: The properties of conducting tetracyanoquinodimethane Langmuir-Blodgett films: A study using acoustoelectric devices, Thin Solid Films 160 (1988) 53.

[10] Vikholm, I., Helle H.: Characterization of n,n'-Diarachidoylindigo transferred onto a surface acoustic wave device, Thin Solid Films 178 (1989) 197-202.

[11] Ricco A.J., Staton, A.W., Crooks, R.M., Kim T.: Single-monolayer in situ modulus measurements using a SAW device, Farady Discuss. 107 (1997) 247-258.

[12] Matson J.E., Nguyen T.H., Lynnworth L.C.: Ultrasonic measurement of liquid flow using clamp-on Rayleigh wave transducers, Proc. IMTC-IEEE Instrumentation and Measurement Technology Conference, Boston, MA, 1987, 197-206.

[13] Deighton M.O., Gillespie A.B., Pike R.B., Watkins R.D.: Mode conversion of Rayleigh and Lamb waves to compression waves at a metal-liquid interface, Ultrasonics 19 (1981) 249-258.

[14] Lindner G., Faustmann H., Frankenberger S., Münch M., Pflaum K., Rothballer S., Unterburger $M$.: A versatile acoustic waveguide sensor for liquids based on multiple mode conversion at solidliquid interfaces, Proc. IEEE Ultrasonic Symposium, Vancouver, 2006, 1181-1184.

[15] Lindner G.: Sensors and actuators based on surface acoustic waves propagating along solidliquid interfaces, J. Phys. D: Appl. Phys. 41 (2008) 123002 (13p.).

[16] Faustmann H., Münch M., Markert W., Lindner G.: Acoustic liquid sensor based on Lamb waves on structural materials, Proc. Eurosensors XXII, Dresden, 2008, 227-230.

[17] Nayfeh A.H., Chimenti, D.E., Adler, L., Crane R.L.: Ultrasonic leaky waves in the presence of a thin layer, J. Appl. Phys. 52 (1981) 4985-4994.

[18] Chimenti, D.E., Nayfeh, A.H., Butler, D.L.: Leaky Rayleigh waves on a layered halfspace, J. Appl. Phys. 53 (1982) 170-176. 